\title{
Bericht von der 21. Internationalen Arbeitstagung der GTA in Warschau (2019)
}

Von 13. bis 15. Juni 2019 fand in Warschau die 21. wissenschaftliche Arbeitstagung der International Society for Gestalt Theory and its Applications (GTA) statt, die dem Rahmenthema "Motion. Spaces of Human Experience“ gewidmet war. Veranstalter waren die Universität Warschau, vertreten durch Silvia Bonacchi, die Polnische Akademie der Wissenschaften, vertreten durch Jagna Brudzińska in Zusammenarbeit mit der Universität Palermo, vertreten durch Alice Pugliese, die Universität Triest und die von Stanisław Czerniak vertretene Fundacja Filozofia na Rzecz Dialogu. Tagungsort dieser Jubiläumstagung, auf der auch das 40jährige Bestehen der GTA gefeiert wurde, war der Staszic-Palast im Herzen Warschaus und Sitz der Polnischen Akademie der Wissenschaften. Die Tagung hatte einen ausgeprägt interdisziplinären Charakter: Nicht nur Psychologen, sondern Wissenschaftler aus den unterschiedlichsten Disziplinen waren eingeladen, sich mit dem Konzept „Motion“/„Bewegung“ und der Gestalttheorie auseinanderzusetzen.

Die einzelnen Sessions begannen jeweils mit Pleanrvorträgen der Invited Speakers Tiziano Agostini (Universität Triest), Michael Buchholz (International Psychoanalytic University (IPU), Berlin), Angelo Giavatto (Université de Nantes et Centre Atlantique de Philosophie), Elżbieta Jung (Universität Łódź), Michail Kotin (Universität Zielona Góra), Hilge Landweer (FU Berlin) und Christoph Wolf (FU Berlin). Im Rahmen der 40-Jahr-Feier der GTA sprachen deren 1. Vorsitzender Hellmuth Metz-Göckel, Kurt Guss als Mitbegründer der GTA, und der langjährige Herausgeber der Gestalt Theory Gerhard Stemberger. Jeweils im Anschluss an die Plenarvorträge fanden die zahlreichen weiteren Vorträge sowie die drei workshops des Kongresses in einzelnen, nach Themenschwerpunkten zusammengestellten, Sektionen statt. Eine Poster-Session gab am 2. Tag Gelegenheit, sich mit jungen polnischen Wissenschaftlern zu den speziellen Themen ihrer Präsentationen auszutauschen.

Nach der Begrüßung durch die Leiter der einladenden Institutionen Danilo Facca, Vize-Direktor des Instituts für Philosophie und Soziologie der Polnischen Akademie der Wissenschaften, Stanisław Czerniak vom Institut für Philosophie und Soziologie der Polnischen Akademie der Wissenschaften und Elżbieta 
Jamrozik, Direktorin des Instituts für Fachkommunikation und interkulturelle Kommunikation (IKSI) der Universität Warschau fand der erste Plenarvortrag statt. Christoph Wolf (FU Berlin) zeigte in seinem Vortrag „Die Bewegung der Wiederholung. Inkorporierung durch Mimesis, Ritual und Imagination", dass die Bewegung der Wiederholung unaufhebbar mit der Konstitution des menschlichen Körpers verbunden und daher eine conditio humana ist. Dies wird durch den Prozess der Hominisation deutlich, an dem abzulesen ist, dass Wiederholung für die Evolution des homo sapiens und die Entwicklung von Gemeinschaften und Individuen wesentlich ist. Dabei sind Wiederholungen mimetisch sie führen zu produktiven Nachahmungen, in denen auch Neues entsteht. Mimetische Bewegungen helfen dem Menschen, die historische und kulturelle Welt zu erschließen. Die dabei entstehenden Emotionen werden als Bewegungen angesehen, durch die sich eine Orientierung in der Welt vollzieht.

Der zweite Plenarvortrag, gehalten von Michael Buchholz (IPU Berlin), war dem Thema „Movement in Therapeutic Spaces - Seeing the Situational Gestalt of Typical Problematic Situations (TPS)“ gewidmet. Buchholz legte seinen Ausführungen die These von Norcross und Wampold (2018) zugrunde, dass "transdiagnostische Eigenschaften" wie Geschlecht, Alter, Hautfarbe, Religion, Einkommen, gesellschaftliche Schicht u.a. dem Therapeuten einen besseren Ausgangspunkt in seiner Arbeit bieten als schematische diagnostische Klassifikationssysteme wie etwa DSM oder ICD. Daran anschließend wurden ausgewählte Ergebnisse einer Studie von typischen Situationen (Co-Constructed Utterances - CCU; agenda transforming utterances - ATU; typical problematic situations - TPS) präsentiert und Buchholz zeigte, dass diese als Situationsgestalten mit einer rhythmischen Kontur, die man beschreiben könne, vorkommen. Im zweiten Teil seines Vortrags fokussierte der Autor darauf, wie nonverbales Verhalten des Therapeuten das Resultat einer therapeutischen Sitzung beeinflussen kann.

Der zweite Teil dieses ersten Kongressnachmittags war der Feier des 40jährigen Bestehens der veranstaltenden Gesellschaft für Gestalttheorie und ihre Anwendung - GTA gewidmet und gab in drei Vorträgen Einblicke zur Gründungsgeschichte der GTA, zu zentralen wissenschaftlichen Positionen und zu möglichen Standortbestimmungen der Gestalttheorie heute. Hellmuth Metz-Göckel, 1. Vorsitzender der Gesellschaft, eröffnete seinen Vortrag „From ,Prägnanz' to Self-Organization - Central Gestalt-theoretical Constructs“ mit der Feststellung der zentralen gestalttheoretischen Annahme, dass psychische Prozesse nach einer optimalen, ausgewogenen Ordnung streben. Wir sehen eine wohlgeordnete und wohlstrukturierte Welt, wobei Störungen und Irregularitäten möglichst beseitigt werden sollen. Ordnung und Struktur liegen vor, wenn keine Kontrolle oder kein Eingriff seitens der Außenwelt oder der Person selbst stattfinden. Diese Prozesse finden wie von selbst 
statt. Der Autor führte hierfür den Begriff „Prägnanz“ an, wie er von der Gestalttheorie gebraucht wird, plädierte in diesem Kontext aber für den Begriff „Selbstorganisation“, der allerdings Prägnanz mit einschließe, und nannte Beispiele von Selbstorganisation aus der Forschungspraxis.

„Does a Society for Gestalt Theory and its Applications still fit in our time?“ fragte Gerhard Stemberger, ehemaliger 1. Vorsitzender der GTA und langjähriger Herausgeber der Gestalt Theory, in seinem Vortrag. Er stellte Argumente für die These dar, dass es nach wie vor auch heute noch wissenschaftliche Schulen gäbe, auch wenn diese nicht so genannt werden, und dass es gute Gründe für deren Existenz gibt. Nach allgemeinen Überlegungen über das Bestehen von wissenschaftlichen Schulen plädierte der Autor für die Notwendigkeit, Gestalttheorie - als eines von mehreren wissenschaftlichen Systemen -, ebenfalls als eine "Schule“, zu pflegen.

Der dritte Vortrag wurde von Kurt Guss, Mitbegründer der GTA, unter dem Titel „40 years ago - The Foundation Period of the GTA“ gehalten. Guss beschrieb die Situation der Psychologie als wissenschaftlicher Disziplin in der Bundesrepublik Deutschland in den 1970er Jahren, als die Idee entstand, die GTA zu gründen. Psychologie stand damals unter großem Einfluss des Behaviorismus und der Psychoanalyse. Gemeinsam mit dem Verleger Jürgen Steinkopff und dem Psychologen und Psychotherapeuten Hans-Jürgen Walter entstand der Entschluss, eine Organisation zu gründen, die die Forschungen von Max Wertheimer, Kurt Koffka und Wolfgang Köhler weiterverbreiten und Wissenschaftler und Praktiker unterschiedlichster Disziplinen, die in der Tradition der Gestalttheorie der Berliner Schule stehen, zu unterstützen. So wurde 1978 die Gesellschaft für Gestalttheorie und ihre Anwendungen - GTA unter dem Ehrenvorsitz von Wolfgang Metzger, der das Projekt von Anfang an unterstützt hatte, ins Leben gerufen.

Zum Abschluss des ersten Kongresstages trafen sich die Teilnehmer in den Räumen des Palais Staszic zu einem geselligen Beisammensein, wobei auf das Wohl von Prof. Stanisław Czerniak angestoßen wurde und alle Beteiligten die Gelegenheit hatten, miteinander ins Gespräch zu kommen.

Der zweite Tag begann mit dem Plenarvortrag von Michail Kotin (Universität Zielona Góra) „Sein und Bewegung im Spiegel der Sprache: Zur Versprachlichung spatialer und direktionaler Konzepte“. Das Ziel des Vortrags legte der Autor als Versuch fest, die wichtigsten Kodierungsformen von Raum (Spatium) und Bewegung durch Sprachformen zu zeigen. Dabei wird das Konzept „Bewegung“ als ein vom Primitivum „Ort“ abgeleitetes Konzept betrachtet, was an den natürlichen Sprachen deutlich sichtbar ist. Zur Begründung dieser These wurden zahlreiche Beispiele angeführt. 
Im folgenden 2. Pleanrvortrag sprach Tiziano Agostini (Universität Triest) über „Rhythm: A gestalt of human movement". Ausgehend von der Erkenntnis, dass Rhythmus eine Grundlage menschlicher Bewegung bildet, formulierte Agostini auf Grund von Forschungen seines Forschungsteams drei weitere Erkenntnisse: 1. die zyklische Bewegung des menschlichen Körpers kann exakt durch einen akustischen Stimulus wiedergegeben werden. Der Stimulus kann von der Person erkannt werden, die ihn produziert hat. 2. „Ökologische“ Geräusche (Geräusche, die durch den menschlichen Körper produziert werden) sind bei der Herausbildung einer effektiven mentalen Repräsentation einer motorischen Handlung effizienter. 3. Akustische Informationen stellen eine wichtige Rückmeldung für eine bessere Kontrolle des Timings dar, wenn man eine gegebene Bewegung standardisieren oder verbessern will.

Nach den Plenarvorträgen standen den Teilnehmern nach einer kurzen Kaffeepause parallel mehrere Sektionen mit unterschiedlichen thematischen Schwerpunkten zur Auswahl, in denen Referenten über aktuelle Arbeiten berichteten und daran anschließend Diskussionen möglich waren.

Sektion A: Moving through words (Bewegung durch Worte):

ReferentInnen: Savina Raynaud (Mailand): „Moving Words“. Ein Referat über die Rolle der Wortstellung beim Gestalten und Bewegen von Gedanken und Leidenschaften des Adressaten.

Chiara De Vita / Maria Chiara Passolunghi (Triest): „Karl Bühlers fantasmatic deixis between motion, gestures and words“. Eine Studie über den Übergang vom „unmittelbaren“ Handeln („,immediate“ behaviour) zum „mittelbaren“ Handeln (Erinnerungen d.h. Retrospektion bzw. konstruktive Phantasie d.h. Prospektion) im Kontext der Deixis am Phantasma.

Sektion B: Movement and cognition (Bewegung und Erkenntnis):

ReferentInnen: Giulia Parovel / Nicolò Mencucci (Siena) „Comic aftereffects of incongruent causal contingencies”. Ein Referat über bestimmte zeiträumliche Konfigurationen, die durch die Manipulation der perzeptuellen Kausalität und Belebtheit zum Erzielen inkongruenter kausaler Kontingente hervorgerufen werden, als Katalysatoren von gewissen komischen Eindrücken.

Elena Capitani (Referentin, Macerata) / Ivana Bianchi (Macerata) / Roberto Burro (Verona) / Erika Branchini (Verona) / Ugo Savardi (Verona): „What is the opposite of a process? The meaning of ,opposite' in represented spatial processes. “ Ein Referat über zwei Studien zur Messung menschlicher Fähigkeit, mittels Gegensätzen zu schlussfolgern.

Sektion C: Rhythm as gestalt of human movement (Rhythmus als Gestalt der menschlichen Bewegung): 
ReferentInnen: Giulio Baldassi (Referent) / Ilaria Santoro / Serena Mingolo / Mauro Murgia / Tiziano Agostini (Trieste): „Ecological sounds are more effective than artificial sounds in breath modulation". Ein Vergleich natürlicher und künstlicher Geräusche auf die menschliche Atemlänge.

Mauro Murgia (Referent) / Massimiliano Pau / Federica Corona / Roberta Pili / Carlo Casula / Marco Guicciardi / Tiziano Agostini (Trieste): „Rhythmic auditory stimulation and gait rehabilitation in Parkinson's disease: artificial vs ecological cues". Eine Studie über die Ergebnisse der Rehabilitation von Parkinson-Kranken bei Verwendung natürlicher und künstlicher RAS-Signale (rhythmic auditory stimulation).

Die Mittagspause konnte Dank eines hervorragenden Caterings in den prächtigen Räumen des Palais Staszic zwanglos zu Gesprächen und weiterem Austausch genützt werden. Den Übergang zur Nachmittagssitzung bildete eine Postersession, in der folgende Poster präsentiert wurden: Yuliia Savchuk (Lutsk) „Psychological complexes: genesis and work over them" (eine Analyse der Ursprünge psychologischer Komplexe und der Methoden ihrer psychotherapeutischen Verarbeitung), Paweł Kluczek (Warschau) „Motion in communication: borrowings in the gamers' language" (eine Studie über englische Entlehnungen in der deutschen Gamersprache und deren Gebrauch), Aneta Borowiecka / Kamil Böhm / Maja Falkenberg / Klaudia Wielemborek (Warschau) „Movement in communication: The significance of nonverbal language" (Vorstellung des Labors für Multimodale Kommunikation der Universität Warschau und dessen Aktivitäten, speziell anhand der nonverbalen Elemente von Charisma und von Gesten mit Missverständnispotential).

Die Nachmittags-Session begann wieder mit einem Plenarvortrag: Hilge Landweer (Institut für Philosophie der FU Berlin) sprach über „Die Räumlichkeit der Gefühle“. Die aktuell stark diskutierte Theorie der Gefühle und Stimmungen („Atmosphären“) von Hermann Schmitz wurde ausführlich vorgestellt und in der Folge anhand der Begriffe „verbreiten“ und „ausstrahlen“ das wörtliche Verständnis von Gefühlsbeschreibungen, die diese räumlichen Metaphern enthalten, diskutiert.

Nach dem Plenarvortrag folgten hintereinander zwei Blöcke thematisch unterschiedlicher paralleler Sektionen mit Referaten und anschließender Diskussion, an diesem Nachmittag auch ein, der Gestalttheoretischen Psychotherapie (GTP) gewidmeter, workshop (Sektion D).

Sektion A: Expressing Motion (Bewegung ausdrücken)

ReferentInnen: Joanna Pędzisz (Lublin) „,Jede Bewegung verläuft in der Zeit und hat ein Ziel' (Aristoteles). Zum interdisziplinären Blick auf den Diskurs der zeitgenössischen Tänzer". Ein inter- und transdisziplinärer Blick auf den Tanzdiskurs, der die Frage der Versprachlichung eines Tanzereignisses, die Kennzeichnung der 
Körper- und Bewegungskonzepte, die Diskursgemeinschaft der zeitgenössischen Tänzer und die während der Tanzlernprozesse realisierten Sprechakte in den Blick nimmt.

Rafał Augustyn (Lublin) „Expressing motion in multimodal film setting“. Eine Analyse verschiedener Dimensionen der visuell, audio-visuell und verbal kodierten Bewegung in den Filmen „Arrival“ (2016) and „Blade Runner 2049“ (2017).

Sektion B: Motion and cognition (Bewegung und Erkenntnis)

ReferentInnen: Jacqueline Bellon (Siegen) „Grund, Figur und Grenzübergänge als Motoren für Bewegung“. Eine Analyse des Konzeptes „Bewegung“, wie es von dem französischen Philosophen und Psychologen Gilbert Simondon definiert wurde, und dessen Bezug zu den Konzepten „Grund“ und „Figur" als Motoren aller Bewegungsprozesse.

Olha Dunajewska (Kyiv) „Godfather - motionless gains motion (15 shades of Godfather)". Ein gestaltlinguistischer Versuch, am Beispiel des Lexems "godfather" im Rahmen der BAPTISM-Gestalt den Mechanismen der Sprache auf den Grund zu gehen.

Sektion C: Motion in space (Bewegung im Raum)

ReferentInnen: Maciej Talaga (Warschau) „Archaelogy of movement. Historical records and modern bodies as media for investigating". Ein Versuch, Bewegung aufgrund historischer Notation am Beispiel einer mittelalterlichen Abhandlung über Kampfkunst mithilfe methodologischer Kombination textbasierter Forschungen und praktischer Experimente (embodied research) zu rekonstruieren.

Andrea Lanza (Florenz / Polnische Akademie der Wissenschaften) „Kinesthetic unity as motivated association”. Eine Analyse des Konzeptes der „instinktiven“ Spontanität des Egos (Husserl) im Kontext „kausaler“ Motivationsprinzipien.

Sektion D - workshop: „Motion in Gestalt theoretical psychotherapy. Moving through life experience - Gestalt theoretical psychotherapy - part I: Theoretical foundations“ („Bewegung in gestalttheoretischer Psychotherapie. Bewegung durch Lebenserfahrung - gestalttheoretische Psychotherapie - Teil I):

ReferentInnen: Katharina Sternek „The Epistemic position”. Eine Besprechung der Hauptansätze des kritischen Realismus der Gestalttheorie und dessen praktischen Wertes für das Verständnis der Dynamik zwischenmenschlicher Beziehungen und der Gestaltung der Beziehung in einer Psychotherapie.

Bernadette Lindorfer "Personality Theory”. Eine Besprechung von Lewins Konzept „Spannung” als gestalttheoretische Entsprechung des Konzeptes „Triebtheorie“ von Freud. 
Angelika Böhm „Basic principles for the therapeutic relationship and practice”. Eine Analyse des Konzeptes „Kennzeichen der Arbeit am Lebendigen” von Wolfgang Metzger (1962) im Kontext therapeutischer Beziehung und Praxis mit dem Zweck, das therapeutische Umfeld als Ort kreativer Freiheit zu gestalten.

Der zweite Block der Sektionen und des workshops schloss direkt an.

Sektion A: Motion, expression and emotion (Bewegung, Ausdruck und Emotion):

ReferentInnen: Luca Taddio / Damiano Cantone (Udine) „Merleau-Ponty: From the Psychology of Gestalt to the Analysis of Movement and Cinema”. Eine Besprechung des Begriffs der „scheinbaren Bewegung” Merleau-Pontys und dessen Bezug zu Ergebnissen gestalttheoretischer Experimente sowie der Versuch, am Beispiel einer Filmmontage zu entscheiden, ob der Begriff „scheinbar“ tatsächlich den Sachverhalt trifft oder ob ontologische Kategorien besser zur Beschreibung des Sachverhalts geeignet wären.

Mauro Antonelli (Mailand): „Vittorio Benussi and "Emotional Functional Autonomy'. Replication and Re-evaluation”. Eine kritische Betrachtung der Forschung Vittorio Benussis zur "funktionellen emotionalen Autonomie“ aus den 1920er Jahren, die von hypnosuggestiven Methoden Gebrauch machte.

Sektion B: Movement and Arts (Bewegung und Künste)

ReferentInnen: Herbert Fitzek (Berlin): „ART moves MIND moves ART - The Moses of Michelangelo and the Gestaltkreis of Art Reception”. Ein psychologischer Blick auf Kunst als ästhetisches Handlungsfeld, wo Kunstwerke keine statischen Gegenstände und Rezipienten keine passiven Zuschauer sind, mit Bezug auf Victor von Weizsäckers Gestaltkreis der Wahrnehmung und Handlung, analysiert am Beispiel von Michelangelos „Der Moses“.

Mitchell Atkinson III (Polnische Akademie der Wissenschaften): „Spaces of Possibility and their Spontaneous Sonic Exploration: Toward a Phenomenology of Improvised Music“. Ein Versuch, mit Hilfe der Phänomenologie musikalische Improvisation als spontane Entdeckung prädeterminierter Möglichkeitsräume zu beschreiben.

Sektion C: Movement in action (Bewegung im Einsatz)

ReferentInnen: Alicja Waszkiewicz-Raviv (Warschau): „Gestalt Public Relations. Perception laws as sequencing theory for organizational communication activities". Ein Versuch, gestalttheoretische Ansätze im Kontext der Public Relations zu betrachten.

Reinhold Utri (Warschau): „Mens sana in corpore sano - Learning and Movement. Effects of physical movement before and during the learning process". Ein 
Ansatz, die positive Wirkung der Bewegung auf die Kognition unter Beweis zu stellen.

Nach einer kurzen Kaffeepause wurden den Teilnehmern zum Abschluss des 2. Kongresstages drei thematisch unterschiedliche workshops angeboten:

Workshop 1: Monika Płużyczka / Agnieszka Andrychowicz-Trojanowska / Anna Bonek / Maria Nikishina / Dominik Kudła (Warschau): „Can these eyes lie? Eye tracking in linguistics“. Der Workshop präsentierte die Arbeit des Labors für experimentelle Eye-tracking-Linguistik (LELO) der Universität Warschau und die einzelnen Forschungsprojekte, die in seinem Rahmen realisiert werden (u.a. Analyse der Augenbewegung beim Fremdsprachenlernen aus einem Lehrbuch).

Workshop 2: Chiara Salvatore (Pomezia, Rom): „Creative Lab named HAIKUBOOKLET“. Die Leiterin des Workshops nannte den Workshop „Fitnessstudio” zur Vergrößerung des Realitätsraumes im Gruppenkontext, das ermöglicht, sich selbst zu testen, und sich von der Wahrnehmung bis zum vollen Kontakt zu bewegen.

Workshop 3: Fortsetzung des workshops Gestalttheoretische Psychotherapie: Motion in Gestalt theoretical psychotherapy (Part II: Practice of Gestalt Theoretical Psychotherapy) (Bewegung in gestalttheoretischer Psychotherapie. Teil II: Praxis der gestalttheoretischen Psychotherapie):

Referent: Thomas Fuchs (GTA): „Gestalt Theoretical Psychotherapy - A Clinical Example". Vorgestellt wurde der Fall einer essgestörten Person, die mithilfe der gestalttheoretischen Ansätze behandelt wurde, mit einem Schwerpunkt auf die praktische Umsetzung der theoretischen Konzepte.

Nach diesem sehr erfüllten Kongresstag gab es die Möglichkeit zu einem gemeinsamen Essen aller Teilnehmer, das im Garten eines vorzüglichen italienischen Restaurants wieder zwanglose Begegnungen und auch weiterführende Diskussionen des Tagungsthemas ermöglichte.

Der dritte und letzte Tag begann wieder mit zwei Plenarvorträgen, beginnend mit Elżbieta Jung (Łódź): „Between Ancient and Modern Times: the Theory of Motion in the 14th Century". Jung vollzog den Einfluss des Bewegungskonzepts Aristoteles' auf Englische Mathematiker, Logiker und Philosophen des 14. Jahrhunderts nach, die versucht hatten, die zahlreichen Inkonsistenzen in Aristoteles Theorie zu erklären, und damit Einfluss auf die Entwicklung des Begriffs „beschleunigte Bewegung“ Galileis nahmen.

In seinem direkt anschließenden Plenarvortrag „Kinêsis. Or how to go beyond human limits through movement" stellte Angelo Giavatto (Nantes) Theorien altgriechischer Philosophen über Bewegung als Methode, die Begrenztheit des 
menschlichen Daseins zu überwinden, vor und stellte Bezüge zu gestalttheoretischen Theoremen her.

Nach den beiden Plenarvorträgen fanden die Vorträge wie üblich in drei Sektionen statt:

Sektion A: Motion and intersubjectivity (Bewegung und Intersubjekitivität)

ReferentInnen: Natalia Artemenko (Sankt Petersburg): „Thematisation of Passivity in Husserl's Phenomenology and the Problem of the Intersubjective World“. Eine Erörterung des Begriffs der „passiven Konstitution” Husserls auch in Bezug auf den Begriff „Intersubjektivität“.

Maria Gołębiewska (Polnische Akademie der Wissenschaften): „The Performative Aspects of Metaphor - between Intentionality and Conventionality“. Die Referentin besprach zuerst philosophische Reflexionen über Metapher, um dann mit Bezug auf die Theorie der Sprechakte Austins der Frage auf den Grund zu gehen, inwieweit die Fundierung einer metaphorischen Bedeutung von der sprachlichen Konvention und von der Intention des Sprechers abhängt.

Anna Michalska (Polnische Akademie der Wissenschaften): „Motion and intersubjectivity“. Den Ausgangspunkt des Referats bildete das Prinzip Köhlers vom Jahre 1947, das besagt, dass menschliche kognitive Systeme sich auf dem ontogenetischen und evolutionären Niveau durch progressive Differenzierung entwickeln. Die Referentin unternahm den Versuch, das Prinzip auf Intersubjektivität zu beziehen und zu zeigen, dass objektive und soziale Kognition zwei Seiten des explorativen Verhaltens sind.

Sektion B: Movement in Language (Bewegung in der Sprache)

ReferentInnen: Alfonsina Buoniconto (Salerno): „Intratypological and intertypological variation in motion events encoding. A diachronic account from three Romance varietes". Buoniconto präsentierte und diskutierte die Ergebnisse einer korpusbasierten Studie über sprachliche Ausdrücke von Bewegung in den romanischen Sprachen in Werken aus drei historischen Perioden.

Christopher Mitchell (Grenoble): „Moving towards appropriate prosody“ (Mitautorin: Anda Fournel). Ausgangspunkt der Arbeit Mitchells für die Aneignung einer stimmigen Prosodie beim Erlernen einer Fremdsprache ist die Feststellung, dass wenig Ähnlichkeit zwischen der dynamischen Aneignung der Muttersprache und der eher statischen Aneignung einer Fremdsprache besteht. Beim Fremdsprachenlernen wird die Aneignung des nonverbalen Verhaltens, das für die zu erlernende Sprache typisch ist, sowie - damit zusammenhängend - der richtigen Prosodie, sehr oft vernachlässigt. Mitchell stellte Aufbau und Ergebnisse eines Experiments vor, in dem Künstler und kognitive Wissenschaftler die Rolle der Interaktion zwischen dem Körper der/des Lernenden in Bewegung, seinem Umfeld und einer weiteren Person zwecks einer verbesserten Prosodie untersuchten. 
Maria Paola Tenchini (Brescia): „Words in Motions - Slurs in Indirect Reports“. In den Mittelpunkt ihres Referats stellte Tenchini Slurs als Teil einer Redewiedergabe. Zuerst wurde der Begriff „Slur” als abwertendes Epitheton definiert, mit dem Menschengruppen, häufig aber auch einzelne Personen, wegen ihrer Hautfarbe, Herkunft, Religion oder sexuellen Orientierung missachtet werden. Dann wurde anhand konkreter Beispiele die Frage diskutiert, ob eine Person, die eine Aussage mit einem Slur-Ausdruck wiedergibt, einen Beleidigungsakt begeht. Es wurde gezeigt, dass sowohl positive als auch negative Antworten möglich sind.

Sektion C: Movement and dance (Bewegung und Tanz)

ReferentInnen: Serena Cattaruzza / Walter Coppola (Triest): „Gestalt and Movement between music and dance". Den Ausgangspunkt des Referats stellte der Aufsatz „Über Gestaltqualitäten” von Christian von Ehrenfels (1890) dar, in dem Gestalt als etwas konkret Hörbares und Sichtbares und nicht als formale Abstraktion verstanden wird. Ehrenfels demonstrierte diese Auffassung am Beispiel einer Melodie: sie ist eine Abfolge von Tönen, die als Einheit neuer Ordnung zu betrachten ist und sich von der reinen Summe ihrer einzelnen Elemente (Töne) unterscheidet. Die Referenten stellten dann die Frage, was es bedeutet, sich eine Melodie zu merken - bedeutet es, sich die Summe oder die Abfolge von Tönen zu merken? Die Frage wurde aus mehreren Perspektiven (auch am Beispiel von Tanz) diskutiert.

Serena Mingolo (Triest): „The perception of temporal deviations in tap dance: The role of experience and modality" (Mitautoren/-innen: Eleonora Bilotta (Calabria) / Giulio Baldassi (Triest) / Tiziano Agostini (Triest) / Mauro Murgia (Triest)): Der Vortrag stellte die Ergebnisse einer Studie vor, in der Experten und Nichtexperten die Aufgabe gehabt hatten, temporale Abweichungen in Stepptanzsequenzen zu entdecken. Die Abweichungen wurden, wie die Autoren erwartet hatten, leichter durch die auditive als die visuelle Modalität entdeckt.

Irene Candelieri (Triest): „Sound, dance and motion in Franz Boas's field research in British Columbia”. Ausgangspunkt des Vortrags war ein Briefzitat des bedeutenden (deutsch)-amerikanischen Ethnologen und Kulturanthropologen Franz Boas, in dem er auf die motorische und gestische Variabilität bei Menschen verschiedener Kulturen hinweist. Boas plädierte für eine holistische Wahrnehmung von Sprache, Musik und Tanz und misst diesen drei kulturellen Phänomenen einen gemeinsamen Nenner, den Rhythmus, zu.

Zum Ende des Kongressprogramms wurde auch dieses Jahr der von der GTA gestiftete Wolfgang-Metzger-Preis für herausragende wissenschaftliche Arbeiten mit gestalttheoretischem Bezug verliehen. In diesem Jahr wurden zwei Wolfgang-Metzger-Preise vergeben und damit zwei Arbeiten ausgezeichnet, die beide von einer Jury als preiswürdig ausgewählt worden waren. Michael Stadler präsentierte in seinem Preisträgervortrag "The Ontological Nature of 
Part-Whole-Oscillations: An Interdisciplinary Determination" eine Alternative zu bisherigen Ansätzen der philosophischen Ontologie, die im Falle von zusammengesetzten Strukturen entweder den Teilen oder dem Ganzen Vorrang geben. Stadler schlägt eine neue ontologische Kategorie „part-whole-oscillation” (pwo) vor und besprach diese detailliert, auch in Bezug auf andere Ansätze aus der Philosophie und der kognitiven Linguistik.

Dirk Paul Bogner, ebenfalls Metzger-Preisträger 2019, hat sich umfassend mit dem Werk Kurt Lewins auseinandergesetzt und wies in seinem Preisträgervortrag „Kurt Lewin's field theory: a forgotten theory in education sciences” darauf hin, dass Lewins Feldtheorie zu Unrecht in Vergessenheit geraten ist. Obwohl die Feldtheorie heute Grundlage zahlreicher wissenschaftlicher oder praxisorientierter Verfahren bildet, sind doch viele ursprüngliche Texte und vor allem damit verbundene Lewinsche Konzepte sowohl in die Wissenschaft als auch die Praxis entweder nur rudimentär oder gar nicht eingeflossen. Bogner zeigte auf, wie die Feldtheorie erfolgreich in der Erziehungswissenschaft praktische Anwendung finden kann.

Erfreulicherweise konnten heuer auch wieder drei Nachwuchs-Förderpreise für wissenschaftliche Abschlussarbeiten vergeben werden, die Ausgezeichneten waren Jacqueline Bellon, Irit Katsur, Cynthia Schroff-Spiering, denen der 1. Vorsitzende Hellmuth Metz-Göckel die Würdigungsurkunden überreichte.

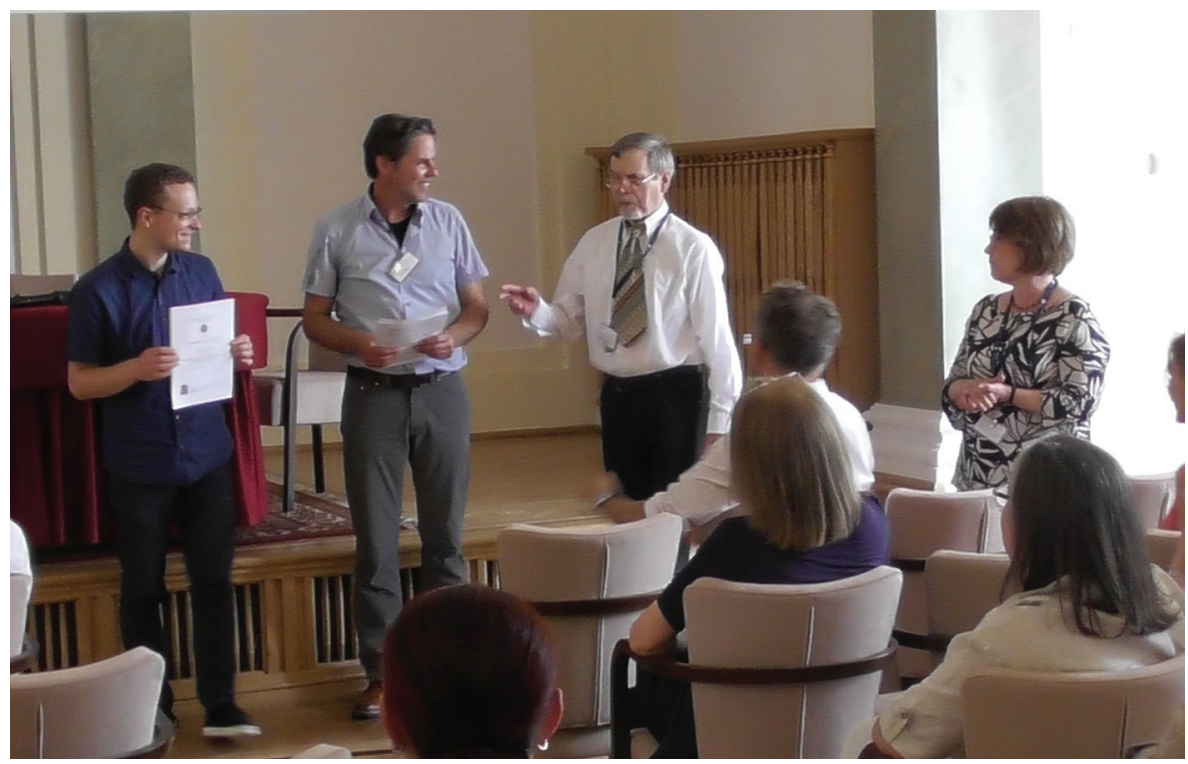

Abb. 1: Die Preisträger des Wolfgang-Metzger-Preises 2019, Michael W. Stadler und Dirk Paul Bogner, bei der Preisverleihung durch den GTA-Vorsitzenden Hellmuth Metz-Göckel. 
Eine besondere Ehrung stand am Ende der diesjährigen wissenschaftlichen Arbeitstagung, die Verleihung der Ehrenmitgliedschaft der GTA an ihr Gründungsmitglied Prof. Dr. mult. Kurt Guss. Der Geehrte bedankte sich mit einem Vortrag über „Kopernikus - Versuch über die Sternstunden des Geistes". Guss führte aus, dass die antiken Seeleute die ersten waren, die sich dessen bewusst geworden sind, dass unsere Wahrnehmung nicht die Wirklichkeit repräsentiert. Diese Erkenntnis nutzten die antiken Philosophen wie z.B. Anaxagoras, der folgerte, dass die Bewegung eines Objekts sich immer aus seiner Beziehung zu einem anderen Objekt ergibt. In diesem Kontext kann auch das heliozentrische Weltbild von Kopernikus angeführt werden, in dem sich die Planeten rund um die Sonne bewegen. Den nächsten Schritt in dieser langen Tradition der Beobachtung der antiken Seeleute bildet nach Guss Kants „Die Kritik der reinen Vernunft" und in einem weiteren Schritt die Gestalttheorie Wertheimers. Alle diese Schritte seien „Sternstunden des Geistes“. Zum Abschluß wurde den Organisatorinnen Silvia Bonacchi, Jagna Brudzińska und Alice Pugliese ausdauernd für ihre erfolgreiche unermüdliche Arbeit applaudiert! Neben dem dichten wissenschaftlichen Programm hatten sie auch das Rahmenprogramm, das von den Teilnehmern ausgiebig genützt worden war, perfekt organisiert und so den Kongress insgesamt zu einem schönen Erfolg geführt. Als kleines Dankeschön wurden namens der GTA Blumen überreicht.

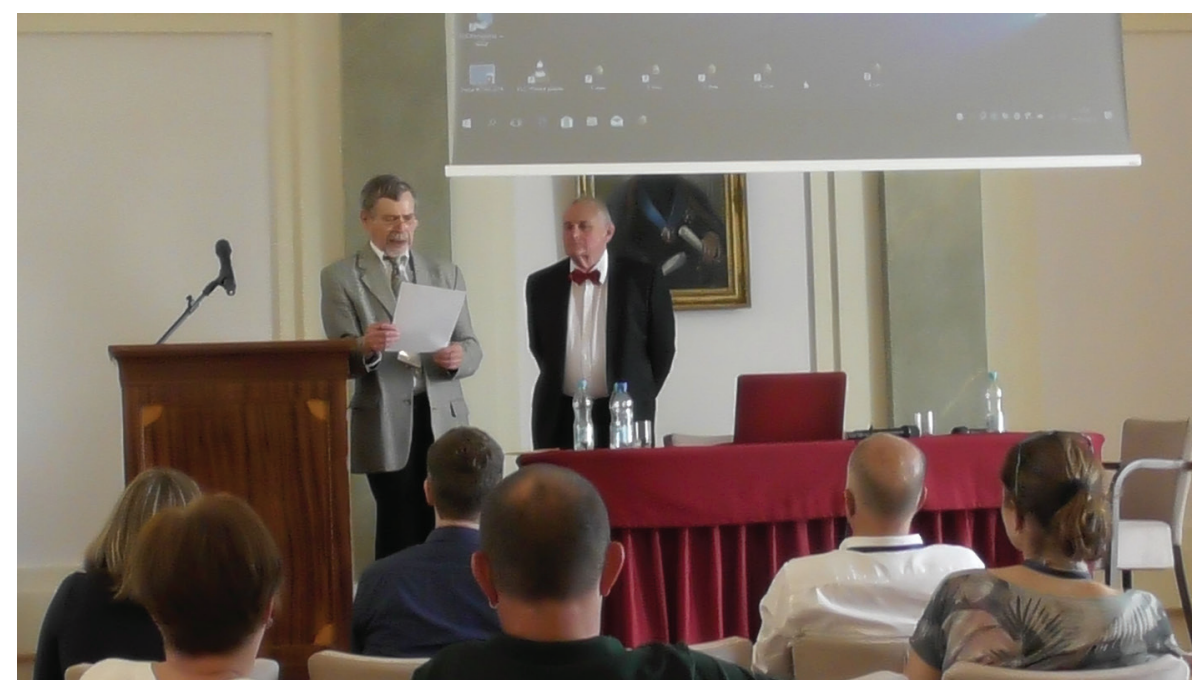

Abb. 2: Kurt Guss wird vom GTA-Vorsitzenden Hellmuth Metz-Göckel die Ehrenmitgliedschaft der GTA verliehen. 


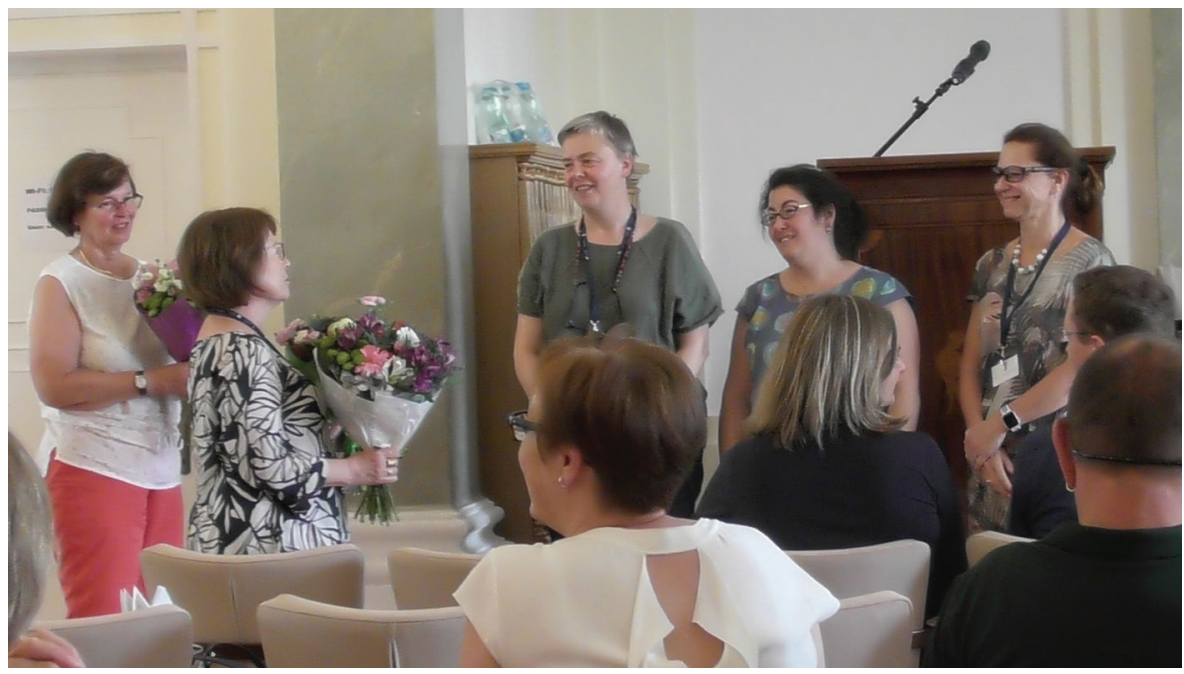

Abb. 3: Die Organisatorinnen (von rechts nach links): Silvia Bonacchi, Alice Pugliese, Jagna Brudzińska bekommen Blumen als Danksagung von den GTA-Mitgliedern Marianne Soff und Bettina Turi-Ostheim.

Am Abend fanden noch eine Stadtführung, geleitet von Agnieszka Dickel von der Universität Warschau, und ein gemeinsames Abendessen an der Weichsel statt. Die Tagung war ein großer Erfolg und die Gäste haben den Wunsch geäußert, sich beim nächsten Kongress wieder zu treffen.

Weitere Informationen über den Kongress, die Referenten/-innen und die erweiterten Abstracts ihrer Referate kann man auf der Internetseite des Kongresses unter www.motion.iksi.uw.edu.pl abrufen. Die Veröffentlichung der Beiträge ist 2020 in drei Heften der Gestalt Theory geplant.

Mariusz Mela, Warschau 